\title{
DETERMINANTS OF PSEUDO-LAPLACIANS
}

\author{
Tayeb Aissiou, luc Hillairet and Alexey Kokotov
}

\begin{abstract}
We derive comparison formulas relating the zeta-regularized determinant of an arbitrary self-adjoint extension of the Laplace operator with domain $C_{c}^{\infty}(X \backslash\{P\}) \subset$ $L_{2}(X)$ to the zeta-regularized determinant of the Laplace operator on $X$. Here, $X$ is a compact Riemannian manifold of dimension 2 or $3 ; P \in X$.
\end{abstract}

\section{Introduction}

Let $X_{d}$ be a complete Riemannian manifold of dimension $d \geq 2$ and let $\Delta$ be the (positive) Laplace operator on $X_{d}$. Choose a point $P \in X_{d}$ and consider $\Delta$ as an unbounded symmetric operator in the space $L_{2}\left(X_{d}\right)$ with domain $C_{c}^{\infty}\left(X_{d} \backslash\{P\}\right)$. It is well known that thus obtained operator is essentially self-adjoint if and only if $d \geq 4$. In case $d=2,3$, it has deficiency indices $(1,1)$ and there exists a one-parameter family $\Delta_{\alpha, P}$ of its self-adjoint extensions (called pseudo-laplacians; see [3]). One of these extensions (the Friedrichs extension $\Delta_{0, P}$ ) coincides with the self-adjoint operator $\Delta$ on $X_{d}$. In case $X_{d}=R^{d}, d=2,3$ the scattering theory for the pair $\left(\Delta_{\alpha, P}, \Delta\right)$ was extensively studied in the literature (see, e.g., [1]). The spectral theory of the operator $\Delta_{\alpha, P}$ on compact manifolds $X_{d}(d=2,3)$ was studied in [3], notice also a recent paper [15] devoted to the case, where $X_{d}$ is a compact Riemann surface equipped with Poincaré metric.

The zeta-regularized determinant of Laplacian on a compact Riemannian manifold was introduced in [11] and since then was studied and used in an immense number of papers in string theory and geometric analysis, for our future purposes we mention here the memoir [5], where the determinant of Laplacian is studied as a functional on the space of smooth Riemannian metrics on a compact two-dimensional manifold, and the papers $[6,13]$, where the reader may find explicit calculation of the determinant of Laplacian for three-dimensional flat tori and for the sphere $S^{3}$ (respectively).

The main result of the present paper is a comparison formula relating $\operatorname{det}\left(\Delta_{\alpha, P}-\right.$ $\lambda)$ to $\operatorname{det}(\Delta-\lambda)$, for $\lambda \in \mathbb{C} \backslash\left(\operatorname{Spectrum}(\Delta) \cup \operatorname{Spectrum}\left(\Delta_{\alpha, P}\right)\right)$ (see Theorem 1 in Section 4 and Theorem 2 in Section 5).

It should be mentioned that in case of two-dimensional manifolds, the zeta regularization of $\operatorname{det}\left(\Delta_{\alpha, P}-\lambda\right)$ is not that standard, since the corresponding operator zeta-function has logarithmic singularity at 0 .

It should be also mentioned that in the case when the manifold $X_{d}$ is flat in a vicinity of the point $P$ we deal with a very special case of the situation (Laplacian on a manifold with conical singularity) considered in [8-10] and, via other method, in [7]. The general scheme of the present work is close to that of [7], although some calculations from [9] also appear very useful for us.

Received by the editors March 5, 2012. 


\section{Pseudo-Laplacians, Krein formula and scattering coefficient}

Let $X_{d}$ be a compact Riemannian manifold of dimension $d=2$ or $d=3 ; P \in X_{d}$ and $\alpha \in[0, \pi)$. Following Colin de Verdière [3], introduce the set

$$
\begin{gathered}
\mathcal{D}\left(\Delta_{\alpha, P}\right)=\left\{f \in H^{2}\left(X_{d} \backslash\{P\}\right): \exists c \in \mathbb{C}: \text { in a vicinity of } P\right. \text { one has } \\
\left.f(x)=c\left(\sin \alpha \cdot G_{d}(r)+\cos \alpha\right)+o(1) \text { as } r \rightarrow 0\right\},
\end{gathered}
$$

where

$$
H^{2}\left(X_{d} \backslash\{P\}\right)=\left\{f \in L_{2}\left(X_{d}\right): \exists C \in \mathbb{C}: \Delta f-C \delta_{P} \in L_{2}\left(X_{d}\right)\right\},
$$

$r$ is the geodesic distance between $x$ and $P$ and

$$
G_{d}(r)= \begin{cases}\frac{1}{2 \pi} \log r, & d=2, \\ -\frac{1}{4 \pi r}, & d=3 .\end{cases}
$$

Then (see [3]) the self-adjoint extensions of symmetric operator $\Delta$ with domain $C_{c}^{\infty}\left(X_{d} \backslash\{P\}\right)$ are the operators $\Delta_{\alpha, P}$ with domains $\mathcal{D}\left(\Delta_{\alpha, P}\right)$ acting via $u \mapsto \Delta u$. The extension $\Delta_{0, P}$ coincides with the Friedrichs extension and is nothing but the self-adjoint Laplacian on $X_{d}$.

Let $R(x, y ; \lambda)$ be the resolvent kernel of the self-adjoint Laplacian $\Delta$ on $X_{d}$.

Following [3] define the scattering coefficient $F(\lambda ; P)$ via

$$
-R(x, P ; \lambda)=G_{d}(r)+F(\lambda ; P)+o(1)
$$

as $x \rightarrow P$. (Note that in [3] the resolvent is defined as $(\lambda-\Delta)^{-1}$, whereas for us it is $(\Delta-\lambda)^{-1}$. This results in the minus sign in (2.2).)

As it was already mentioned the deficiency indices of the symmetric operator $\Delta$ with domain $C_{c}^{\infty}\left(X_{d} \backslash\{P\}\right)$ are $(1,1)$, therefore, one has the following Krein formula (see, e.g., [1], p. 357) for the resolvent kernel, $R_{\alpha}(x, y ; \lambda)$, of the self-adjoint extension $\Delta_{\alpha, P}:$

$$
R_{\alpha}(x, y ; \lambda)=R(x, y ; \lambda)+k(\lambda ; P) R(x, P ; \lambda) R(P, y ; \lambda)
$$

with some $k(\lambda ; P) \in \mathbb{C}$.

The following Lemma relates $k(\lambda ; P)$ to the scattering coefficient $F(\lambda ; P)$.

Lemma 1. One has the relation

$$
k(\lambda ; P)=\frac{\sin \alpha}{F(\lambda ; P) \sin \alpha-\cos \alpha} .
$$

Proof. Send $x \rightarrow P$ in $(2.3)$, observing that $R_{\alpha}(\cdot, y ; \lambda)$ belongs to $\mathcal{D}\left(\Delta_{\alpha, P}\right)$, make use of (2.1) and (2.2), and then compare the coefficients near $G_{d}(r)$ and the constant terms in the asymptotical expansions at the left and at the right.

It follows in particular from the Krein formula that the difference of the resolvents $\left(\Delta_{\alpha, P}-\lambda\right)^{-1}-(\Delta-\lambda)^{-1}$ is a rank one operator. The following simple Lemma is the key observation of the present work.

Lemma 2. One has the relation

$$
\operatorname{Tr}\left(\left(\Delta_{\alpha, P}-\lambda\right)^{-1}-(\Delta-\lambda)^{-1}\right)=\frac{F_{\lambda}^{\prime}(\lambda ; P) \sin \alpha}{\cos \alpha-F(\lambda ; P) \sin \alpha} .
$$


Proof. One has

$$
-F_{\lambda}^{\prime}(\lambda ; P)=\left.\frac{\partial R(y, P ; \lambda)}{\partial \lambda}\right|_{y=P}=\lim _{\mu \rightarrow \lambda} \frac{R(y, P ; \mu)-R(y, P ; \lambda)}{\mu-\lambda} .
$$

Using resolvent identity, we rewrite the last expression as

$$
\left.\lim _{\mu \rightarrow \lambda} \int_{X_{d}} R(y, z ; \mu) R(P, z ; \lambda) d z\right|_{y=P}=\int_{X_{d}}[R(P, z ; \lambda)]^{2} d z .
$$

From (2.3) it follows that

$$
[R(P, z ; \lambda)]^{2}=\left.\frac{1}{k(\lambda ; P)}\left(R_{\alpha, P}(x, z ; \lambda)-R(x, z ; \lambda)\right)\right|_{x=z} .
$$

This implies

$$
-F_{\lambda}^{\prime}(\lambda ; P)=\frac{1}{k(\lambda, P)} \operatorname{Tr}\left(\left(\Delta_{\alpha, P}-\lambda\right)^{-1}-(\Delta-\lambda)^{-1}\right),
$$

which, together with Lemma 1, imply (2.5).

Introduce the domain

$$
\Omega_{\alpha, P}=\mathbb{C} \backslash\left\{\lambda-i t, \lambda \in \operatorname{Spectrum}(\Delta) \cup \operatorname{Spectrum}\left(\Delta_{\alpha, P}\right) ; t \in(-\infty, 0]\right\} .
$$

Then in $\Omega_{\alpha, P}$ one can introduce the function

$$
\tilde{\xi}(\lambda)=-\frac{1}{2 \pi i} \log (\cos \alpha-F(\lambda ; P) \sin \alpha)
$$

(It should be noted that the function $\xi=\Re(\tilde{\xi})$ is the spectral shift function of $\Delta$ and $\left.\Delta_{\alpha, P}.\right)$ One can rewrite $(2.5)$ as

$$
\operatorname{Tr}\left(\left(\Delta_{\alpha, P}-\lambda\right)^{-1}-(\Delta-\lambda)^{-1}\right)=2 \pi i \tilde{\xi}^{\prime}(\lambda) .
$$

\section{Operator zeta-function of $\Delta_{\alpha, P}$}

Denote by $\zeta(s, A)$ the zeta-function

$$
\zeta(s, A)=\sum_{\mu_{k} \in \operatorname{Spectrum}(A)} \frac{1}{\mu_{k}^{s}}
$$

of the operator $A$. (We assume that the spectrum of $A$ is discrete and does not contain 0.)

Take any $\tilde{\lambda}$ from $\left.\mathbb{C} \backslash\left(\operatorname{Spectrum}\left(\Delta_{\alpha, P}\right) \cup \operatorname{Spectrum}(\Delta)\right)\right)$. From the results of [3] it follows that the function $\zeta\left(s, \Delta_{\alpha, P}-\tilde{\lambda}\right)$ is defined for sufficiently large $\Re s$. It is well known that $\zeta(s, \Delta-\tilde{\lambda})$ is meromorphic in $\mathbb{C}$.

The proof of the following lemma coincides verbatim with the proof of Proposition 5.9 from [7].

Lemma 3. Suppose that the function $\tilde{\xi}^{\prime}(\lambda)$ from (2.7) is $O\left(|\lambda|^{-1}\right)$ as $\lambda \rightarrow-\infty$. Let $-C$ be a sufficiently large negative number and let $c_{\tilde{\lambda}, \epsilon}$ be a contour encircling the 
cut $c_{\tilde{\lambda}}$ which starts from $-\infty+0 i$, follows the real line till $-C$ and then goes to $\tilde{\lambda}$ remaining in $\Omega_{\alpha, P}$. Assume that $\operatorname{dist}\left(z, c_{\tilde{\lambda}}\right)=\epsilon$ for any $z \in c_{\tilde{\lambda}, \epsilon}$. Let also

$$
\zeta_{2}(s)=\int_{c_{\tilde{\lambda}, \epsilon ; 2}}(\lambda-\tilde{\lambda})^{-s} \tilde{\xi}^{\prime}(\lambda) d \lambda,
$$

where the the integral at the right-hand side is taken over the part $c_{\tilde{\lambda}, \epsilon ; 2}$ of the contour $c_{\tilde{\lambda}, \epsilon}$ lying in the half-plane $\{\lambda: \Re \lambda>-C\}$. Let

$$
\hat{\zeta}_{2}(s)=\lim _{\epsilon \rightarrow 0} \zeta_{2}(s)=2 i \sin (\pi s) \int_{-C}^{\tilde{\lambda}}(\lambda-\tilde{\lambda})_{0}^{-s} \tilde{\xi}^{\prime}(\lambda) d \lambda,
$$

where $(\lambda-\tilde{\lambda})_{0}^{-s}=e^{-i \pi s} \lim _{\lambda \downarrow c_{\tilde{\lambda}}}(\lambda-\tilde{\lambda})^{-s}$. Then the function

$$
\left.R(s, \tilde{\lambda})=\zeta\left(s, \Delta_{\alpha, P}-\tilde{\lambda}\right)\right)-\zeta(s, \Delta-\tilde{\lambda})-2 i \sin (\pi s) \int_{-\infty}^{-C}|\lambda|^{-s} \tilde{\xi}^{\prime}(\lambda) d \lambda-\hat{\zeta}_{2}(s)
$$

can be analytically continued to $\Re s>-1$ with $R(0, \tilde{\lambda})=R_{s}^{\prime}(0, \tilde{\lambda})=0$.

For completeness, we give a sketch of proof.

Proof. Using (2.7), one has for sufficiently large $\Re s$

$$
\begin{aligned}
\zeta\left(s, \Delta_{\alpha, P}-\tilde{\lambda}\right)-\zeta(s, \Delta-\tilde{\lambda}) & =\frac{1}{2 \pi i} \int_{c_{\tilde{\lambda}, \epsilon}}(\lambda-\tilde{\lambda})^{-s} \operatorname{Tr}\left(\left(\Delta_{\alpha, P}-\lambda\right)^{-1}-(\Delta-\lambda)^{-1}\right) d \lambda \\
& =\int_{c_{\tilde{\lambda}, \epsilon}}(\lambda-\tilde{\lambda})^{-s} \tilde{\xi}^{\prime}(\lambda) d \lambda=\zeta_{1}(s)+\zeta_{2}(s),
\end{aligned}
$$

where

$$
\zeta_{1}(s)=\left\{\int_{-\infty+i \epsilon}^{-C+i \epsilon}-\int_{-\infty-i \epsilon}^{-C-i \epsilon}\right\}(\lambda-\tilde{\lambda})^{-s} \tilde{\xi}^{\prime}(\lambda) d \lambda .
$$

It is easy to show (see Lemma 5.8 in [7]) that in the limit $\epsilon \rightarrow 0 \zeta_{1}(s)$ gives

$$
2 i \sin (\pi s) \int_{-\infty}^{-C}|\lambda|^{-s} \tilde{\xi}^{\prime}(\lambda) d \lambda+2 i \sin (\pi s) \int_{-\infty}^{-C}|\lambda|^{-s} \tilde{\xi}^{\prime}(\lambda) \rho(s, \tilde{\lambda} / \lambda) d \lambda,
$$

where $\rho(s, z)=(1+z)^{-s}-1$ and

$$
\rho(s, \tilde{\lambda} / \lambda)=O\left(|\lambda|^{-1}\right),
$$

as $\lambda \rightarrow-\infty$. Using the assumption on the asymptotics of $\tilde{\xi}(\lambda)$ as $\lambda \rightarrow-\infty$ and the obvious relation $\rho(0, z)=0$, one can see that the last term in (3.2) can be analytically continued to $\Re s>-1$ and vanishes together with its first derivative with respect to $s$ at $s=0$. Denoting it by $R(s, \tilde{\lambda})$, one gets the Lemma.

As it is stated in the introduction the main object, we are to study in the present paper is the zeta-regularized determinant of the operator $\Delta_{\alpha, P}-\lambda$. Let us remind the reader that the usual definition of the zeta-regularized determinant of an operator $A$

$$
\operatorname{det} A=\exp \left(-\zeta^{\prime}(0, A)\right)
$$

requires analyticity of $\zeta(s, A)$ at $s=0$.

Since the operator zeta-function $\zeta(s, \Delta-\tilde{\lambda}$ ) is regular at $s=0$ (in fact, it is true in case of $\Delta$ being an arbitrary elliptic differential operator on any compact manifold) 
and the function $\hat{\zeta}_{2}(s)$ is entire, Lemma 3 shows that the behavior of the function $\zeta\left(s, \Delta_{\alpha, P}-\tilde{\lambda}\right)$ at $s=0$ is determined by the properties of the analytic continuation of the term

$$
2 i \sin (\pi s) \int_{-\infty}^{-C}|\lambda|^{-s} \tilde{\xi}^{\prime}(\lambda) d \lambda
$$

in (3.1). These properties in their turn are determined by the asymptotical behavior of the function $\tilde{\xi}^{\prime}(\lambda)$ as $\lambda \rightarrow-\infty$.

It turns out that the latter behavior depends on dimension $d$. In particular, in the next section, we will find out that in case $d=2$ the function $\zeta\left(s, \Delta_{\alpha, P}-\tilde{\lambda}\right)$ is not regular at $s=0$; therefore, in order to define $\operatorname{det}\left(\Delta_{\alpha, P}-\tilde{\lambda}\right)$ one has to use a modified version of (3.3).

\section{Determinant of pseudo-Laplacian on two-dimensional compact manifold}

Let $X$ be a two-dimensional Riemannian manifold, then introducing isothermal local coordinates $(x, y)$ and setting $z=x+i y$, one can write the area element on $X$ as

$$
\rho^{-2}(z)|d z|^{2} \text {. }
$$

The following estimate of the resolvent kernel, $R\left(z^{\prime}, z ; \lambda\right)$, of the Laplacian on $X$ was found by Fay (see [5]; Theorem 2.7 on page 38 and the formula preceding Corollary 2.8 on page 39 ; note that Fay works with negative Laplacian, so one has to take care of signs when using his formulas).

Lemma 4 (J. Fay). The following equality holds true

$$
\begin{aligned}
\left.-R\left(z, z^{\prime} ; \lambda\right)\right)= & G_{2}(r)+O(r)+\frac{1}{2 \pi}\left[\gamma+\log \frac{\sqrt{|\lambda|+1}}{2}\right. \\
& \left.-\frac{1}{2(|\lambda|+1)}\left(1+\frac{4}{3} \rho^{2}(z) \partial_{z \bar{z}}^{2} \log \rho(z)\right)+\hat{R}\left(z^{\prime}, z ; \lambda\right)\right],
\end{aligned}
$$

where $O(r)$ is $\lambda$-independent, $\hat{R}\left(z^{\prime}, z ; \lambda\right)$ is continuous for $z^{\prime}$ near $z$,

$$
\hat{R}(z, z ; \lambda)=O\left(|\lambda|^{-2}\right)
$$

uniformly with respect to $z \in X$ as $\lambda \rightarrow-\infty ; r=\operatorname{dist}\left(z, z^{\prime}\right)$, $\gamma$ is the Euler constant.

Using (4.1), we immediately get the following asymptotics of the scattering coefficient $F(\lambda, P)$ as $\lambda \rightarrow-\infty$ :

$$
\begin{aligned}
F(\lambda, P)= & \frac{1}{4 \pi} \log (|\lambda|+1)+\frac{\gamma-\log 2}{2 \pi} \\
& -\frac{1}{4 \pi(|\lambda|+1)}\left[1+\left.\frac{4}{3} \rho^{2}(z) \partial_{z \bar{z}}^{2} \log \rho(z)\right|_{z=z(P)}\right]+O\left(|\lambda|^{-2}\right) .
\end{aligned}
$$

Remark 1. It is obvious that the expression $\left.\rho^{2}(z) \partial_{z \bar{z}}^{2} \log \rho(z)\right|_{z=z(P)}$ is independent of the choice of conformal local parameter $z$ near $P$. 
Now from (2.6) and (4.2) it follows that

$$
2 \pi i \tilde{\xi}^{\prime}(\lambda)=-\frac{\frac{1}{4 \pi(|\lambda|+1)}-\frac{b}{(|\lambda|+1)^{2}}+O\left(|\lambda|^{-3}\right)}{\cot \alpha-a-\frac{1}{4 \pi} \log (|\lambda|+1)+\frac{b}{|\lambda|+1}+O\left(|\lambda|^{-2}\right)},
$$

where $a=\frac{1}{2 \pi}(\gamma-\log 2)$ and $b=\frac{1}{4 \pi}\left(1+\frac{4}{3} \rho^{2} \partial_{z \bar{z}}^{2} \log \rho\right)$. This implies that for $-\infty<\lambda \leq-C$, one has

$$
2 \pi i \tilde{\xi}^{\prime}(\lambda)=\frac{1}{|\lambda|(\log |\lambda|-4 \pi \cot \alpha+4 \pi a)}+f(\lambda),
$$

with $f(\lambda)=O\left(|\lambda|^{-2}\right)$ as $\lambda \rightarrow-\infty$. Now knowing (4.3), one can study the behavior of the term (3.4) in (3.1). We have

$$
\begin{aligned}
2 i \sin (\pi s) \int_{-\infty}^{-C}|\lambda|^{-s} \tilde{\xi}^{\prime}(\lambda) d \lambda= & \frac{\sin (\pi s)}{\pi} \int_{-\infty}^{-C}|\lambda|^{-s-1} \frac{d \lambda}{(\log |\lambda|-4 \pi \cot \alpha+4 \pi a)} \\
& +\frac{\sin (\pi s)}{\pi} \int_{-\infty}^{-C}|\lambda|^{-s} f(\lambda) d \lambda .
\end{aligned}
$$

The first integral in the right hand side of (4.4) appeared in ([9], p. 15), where it was observed that it can be easily rewritten through the function

$$
\operatorname{Ei}(z)=-\int_{-z}^{\infty} e^{-y} \frac{d y}{y}=\gamma+\log (-z)+\sum_{k=1}^{\infty} \frac{z^{k}}{k \cdot k !},
$$

which leads to the representation

$$
\begin{aligned}
& \frac{\sin (\pi s)}{\pi} \int_{-\infty}^{-C}|\lambda|^{-s-1} \frac{d \lambda}{(\log |\lambda|-4 \pi \cot \alpha+4 \pi a)} \\
& =-\frac{\sin (\pi s)}{\pi} e^{-s \kappa}[\gamma+\log (s(\log C-\kappa))+e(s)],
\end{aligned}
$$

where $e(s)$ is an entire function such that $e(0)=0 ; \kappa=4 \pi \cot \alpha-4 \pi a$. From this we conclude that

$$
\frac{\sin (\pi s)}{\pi} \int_{-\infty}^{-C}|\lambda|^{-s-1} \frac{d \lambda}{(\log |\lambda|-4 \pi \cot \alpha+4 \pi a)}=-s \log s+g(s),
$$

where $g(s)$ is differentiable at $s=0$.

Now (3.1) and (4.6) justify the following definition.

Definition 1. Let $\Delta_{\alpha, P}$ be the pseudo-Laplacian on a two-dimensional compact Riemannian manifold. Then the zeta-regularized determinant of the operator $\Delta_{\alpha, P}-\tilde{\lambda}$ with $\tilde{\lambda} \in \mathbb{C} \backslash \operatorname{Spectrum}\left(\Delta_{\alpha, P}\right)$ is defined as

$$
\operatorname{det}\left(\Delta_{\alpha, P}-\tilde{\lambda}\right)=\exp \left\{-\left.\frac{d}{d s}\left[\zeta\left(s, \Delta_{\alpha, P}-\tilde{\lambda}\right)+s \log s\right]\right|_{s=0}\right\} .
$$

We are ready to get our main result: the formula relating $\operatorname{det}\left(\Delta_{\alpha, P}-\tilde{\lambda}\right)$ to $\operatorname{det}(\Delta-\tilde{\lambda})$. 
From (3.1), (3.4) it follows that

$$
\begin{aligned}
\frac{d}{d s} & {\left.\left[\zeta\left(s, \Delta_{\alpha, P}-\tilde{\lambda}\right)+s \log s-\zeta(s, \Delta-\tilde{\lambda})\right]\right|_{s=0} } \\
= & \left.\frac{d}{d s} \hat{\zeta}_{2}(s)\right|_{s=0}+\int_{-\infty}^{-C} f(\lambda) d \lambda \\
& \quad-\left.\frac{d}{d s}\left\{\frac{\sin \pi s}{\pi} e^{-s \kappa}[\gamma+\log (s(\log C-\kappa))+e(s)]+s \log s\right\}\right|_{s=0} \\
= & 2 \pi i(\tilde{\xi}(\tilde{\lambda})-\tilde{\xi}(-C))+\int_{-\infty}^{-C} f(\lambda) d \lambda-\gamma-\log (\log C-\kappa) \\
= & \times 2 \pi i \tilde{\xi}(\tilde{\lambda})-\gamma \\
& +\int_{-\infty}^{-C} f(\lambda) d \lambda-2 \pi i \tilde{\xi}(-C)-\log (\log C-4 \pi \cot \alpha+2 \gamma-\log 4) .
\end{aligned}
$$

Note that the expression in the second line of (4.8) should not depend on $C$, so one can send $C$ to $+\infty$ there. Together with (4.2) this gives

$$
\left.\frac{d}{d s}\left[\zeta\left(s, \Delta_{\alpha, P}-\tilde{\lambda}\right)+s \log s-\zeta(s, \Delta-\tilde{\lambda})\right]\right|_{s=0}=2 \pi i \tilde{\xi}(\tilde{\lambda})-\gamma+\log (\sin \alpha /(4 \pi))-i \pi
$$

which implies the comparison formula for the determinants stated in the following theorem.

Theorem 1. Let $d=2$, suppose $\tilde{\lambda}$ does not belong to the union of spectra of $\Delta$ and $\Delta_{\alpha, P}$ and let the zeta-regularized determinant of $\Delta_{\alpha, P}$ be defined as in (4.7). Then one has the relation

$$
\operatorname{det}\left(\Delta_{\alpha, P}-\tilde{\lambda}\right)=-4 \pi e^{\gamma}(\cot \alpha-F(\tilde{\lambda}, P)) \operatorname{det}(\Delta-\tilde{\lambda}) .
$$

Observe now that 0 is the simple eigenvalue of $\Delta$ and, therefore, it follows from Theorem 2 in [3] that 0 does not belong to the spectrum of the operator $\Delta_{\alpha, P}$ and that $\Delta_{\alpha, P}$ has one strictly negative simple eigenvalue when $\alpha \neq 0$. Thus, the determinant in the left-hand side of (4.9) is well defined for $\tilde{\lambda}=0$, whereas the determinant at the right-hand side has the asymptotics

$$
\operatorname{det}(\Delta-\tilde{\lambda}) \sim(-\tilde{\lambda}) \operatorname{det}^{*} \Delta
$$

as $\tilde{\lambda} \rightarrow 0-$. Here $\operatorname{det}^{*} \Delta$ is the modified determinant of an operator with zero mode.

From the standard asymptotics

$$
-R(x, y ; \lambda)=\frac{1}{\operatorname{Vol}(X)} \frac{1}{\lambda}+G_{2}(r)+O(1)
$$

as $\lambda \rightarrow 0$ and $x \rightarrow y$ one gets the asymptotics

$$
F(\lambda, P)=\frac{1}{\operatorname{Vol}(X)} \frac{1}{\lambda}+O(1)
$$

as $\lambda \rightarrow 0$. Now sending $\tilde{\lambda} \rightarrow 0-$ in (4.9) and using (4.10) and (4.11) we get the following corollary of the Theorem 1 . 
Corollary 1. For $\alpha \in(0, \pi)$ the following relation holds true:

$$
\operatorname{det} \Delta_{\alpha, P}=-\frac{4 \pi e^{\gamma}}{\operatorname{Vol}(X)} \operatorname{det}^{*} \Delta .
$$

\section{Determinant of pseudo-Laplacian on three-dimensional manifolds}

Let $X$ be a three-dimensional compact Riemannian manifold. We start with the Lemma describing the asymptotical behavior of the scattering coefficient as $\lambda \rightarrow-\infty$.

Lemma 5. One has the asymptotics

$$
F(\lambda ; P)=\frac{1}{4 \pi} \sqrt{-\lambda}+c_{1}(P) \frac{1}{\sqrt{-\lambda}}+O\left(|\lambda|^{-1}\right),
$$

as $\lambda \rightarrow-\infty$

Proof. Consider Minakshisundaram-Pleijel asymptotic expansion [12]

$$
H(x, P ; t)=(4 \pi t)^{-3 / 2} e^{-d(x, P)^{2} /(4 t)} \sum_{k=0}^{\infty} u_{k}(x, P) t^{k}
$$

for the heat kernel in a small vicinity of $P$, here $d(x, P)$ is the geodesic distance from $x$ to $P$, functions $u_{k}(\cdot, P)$ are smooth in a vicinity of $P$, the equality is understood in the sense of asymptotic expansions. We will make use of the standard relation

$$
R(x, y ; \lambda)=\int_{0}^{+\infty} H(x, y ; t) e^{\lambda t} d t .
$$

Let us first truncate the sum (5.2) at some fixed $k=N+1$, so that the remainder, $r_{n}$, is $O\left(t^{N}\right)$. Defining

$$
\tilde{R}_{N}(x, P ;-\lambda):=\int_{0}^{\infty} r_{n}(t, x, P) e^{t \lambda} d t
$$

we see that

$$
\tilde{R}_{N}(x, P ; \lambda)=O\left(|\lambda|^{-(N+1)}\right)
$$

as $\lambda \rightarrow-\infty$ uniformly with respect to $x$ belonging to a small vicinity of $P$.

Now, for each $0 \leq k \leq N+1$ we have to address the following quantity

$$
R_{k}(x, P ; \lambda):=\frac{u_{k}(x, y)}{(4 \pi)^{3 / 2}} \int_{0}^{\infty} t^{k-\frac{3}{2}} e^{-\frac{d(x, P)^{2}}{4 t}} e^{\lambda t} d t .
$$

According to identity (5.12) below, one has

$$
R_{0}(x, P ; \lambda)=\frac{u_{0}(x, P)}{(4 \pi)^{3 / 2}} \frac{2 \sqrt{\pi}}{d(x, P)} e^{-d(x, P) \sqrt{-\lambda}}=\frac{1}{4 \pi d(x, P)}-\frac{1}{4 \pi} \sqrt{-\lambda}+o(1),
$$

as $d(x, P) \rightarrow 0$. For $k \geq 1$ one has

$$
\begin{aligned}
R_{k}(x, P ; \lambda) & =\frac{u_{k}(x, P)}{(4 \pi)^{3 / 2}} 2^{3 / 2-k}\left(\frac{d(x, P)}{\sqrt{-\lambda}}\right)^{k-1 / 2} K_{k-\frac{1}{2}}(d(x, P) \sqrt{-\lambda}) \\
& =-c_{k}(P) \frac{1}{(\sqrt{-\lambda})^{2 k-1}}+o(1)
\end{aligned}
$$

as $d(x, P) \rightarrow 0$ (see [2], p. 146, f-la 29). Now (5.1) follows from (5.3) to (5.5). 
Now from Lemma 5 it follows that

$$
2 \pi i \tilde{\xi}^{\prime}(\lambda)=-\frac{1}{2 \lambda}+O\left(|\lambda|^{-3 / 2}\right)
$$

as $\lambda \rightarrow-\infty$, therefore, one can rewrite (3.4) as

$$
\frac{\sin (\pi s)}{\pi}\left\{\int_{-\infty}^{-C}|\lambda|^{-s}\left(2 \pi i \tilde{\xi}^{\prime}(\lambda)+\frac{1}{2 \lambda}\right) d \lambda+\frac{C^{-s}}{2 s}\right\},
$$

which is obviously analytic in $\Re s>-\frac{1}{2}$. Thus, it follows from (3.1) that the function $\zeta\left(s, \Delta_{\alpha, P}-\tilde{\lambda}\right)$ is regular at $s=0$ and one can introduce the usual zeta-regularization

$$
\operatorname{det}\left(\Delta_{\alpha, P}-\tilde{\lambda}\right)=\exp \left\{-\zeta^{\prime}\left(0, \Delta_{\alpha, P}-\tilde{\lambda}\right)\right\}
$$

of $\operatorname{det}\left(\Delta_{\alpha, P}-\tilde{\lambda}\right)$.

Moreover, differentiating (3.1) with respect to $s$ at $s=0$ similarly to (4.8) we get

$$
\begin{aligned}
& \left.\frac{d}{d s}\left[\zeta\left(s, \Delta_{\alpha, P}-\tilde{\lambda}\right)-\zeta(s, \Delta-\tilde{\lambda})\right]\right|_{s=0} \\
& \quad=2 \pi i(\tilde{\xi}(\tilde{\lambda})-\tilde{\xi}(-C))+\int_{-\infty}^{-C}\left(2 \pi i \tilde{\xi}^{\prime}(\lambda)+\frac{1}{2 \lambda}\right) d \lambda-\frac{1}{2} \log C,
\end{aligned}
$$

which reduces after sending $-C \rightarrow-\infty$ to

$$
2 \pi i \tilde{\xi}(\tilde{\lambda})+\log \sin \alpha-\log (4 \pi)+i \pi=-\log (\cot \alpha-F(\lambda ; P))-\log (4 \pi)+i \pi,
$$

which implies the following theorem.

Theorem 2. For $d=3$ let $\Delta_{\alpha, P}$ be the pseudo-Laplacian on $X$ and $\tilde{\lambda} \in \mathbb{C} \backslash$ $\left(\operatorname{Spectrum}(\Delta) \cup \operatorname{Spectrum}\left(\Delta_{\alpha, P}\right)\right)$. Then

$$
\operatorname{det}\left(\Delta_{\alpha, P}-\tilde{\lambda}\right)=-4 \pi(\cot \alpha-F(\tilde{\lambda} ; P)) \operatorname{det}(\Delta-\tilde{\lambda}) .
$$

Sending $\tilde{\lambda} \rightarrow 0$ and noting that relation (4.11) holds also in case $d=3$, we get the following corollary.

Corollary 2. For $\alpha \in(0, \pi)$

$$
\operatorname{det} \Delta_{\alpha, P}=-\frac{4 \pi}{\operatorname{Vol}(X)} \operatorname{det}^{*} \Delta .
$$

In what follows, we consider two examples of three-dimensional compact Riemannian manifolds for which there exist explicit expressions for the resolvent kernels: a flat torus and the round (unit) $3 d$-sphere. These manifolds are homogeneous, so, as it is shown in [3], the scattering coefficient $F(\lambda, P)$ is $P$-independent.

\section{Example 1: Round 3d-sphere.}

Lemma 6. Let $X=S^{3}$ with usual round metric. Then there is the following explicit expression for scattering coefficient

$$
F(\lambda)=\frac{1}{4 \pi} \operatorname{coth}(\pi \sqrt{-\lambda-1}) \cdot \sqrt{-\lambda-1}
$$


and, therefore, one has the following asymptotics as $\lambda \rightarrow-\infty$ :

$$
F(\lambda)=\frac{1}{4 \pi} \sqrt{|\lambda|-1}+O\left(|\lambda|^{-\infty}\right) .
$$

Remark 2. The possibility of finding an explicit expression for $F(\lambda)$ for $S^{3}$ was mentioned in [3]. However, we failed to find (5.10) in the literature.

Proof. We will make use the well-known identity (see, e.g., [2], p. 146, f-la 28):

$$
\int_{0}^{+\infty} e^{\lambda t} t^{-3 / 2} e^{-\frac{d^{2}}{4 t}} d t=2 \frac{\sqrt{\pi}}{|d|} e^{-|d| \sqrt{-\lambda}}
$$

for $\lambda<0$ and $d \in \mathbb{R}$ and the following explicit formula for the operator kernel $e^{-t} H(x, y ; t)$ of the operator $e^{-t(\Delta+1)}$, where $\Delta$ is the (positive) Laplacian on $S^{3}$ (see [4], (2.29)):

$$
e^{-t} H(x, y ; t)=-\left.\frac{1}{2 \pi} \frac{1}{\sin d(x, y)} \frac{\partial}{\partial z}\right|_{z=d(x, y)} \Theta(z, t) .
$$

Here, $d(x, y)$ is the geodesic distance between $x, y \in S^{3}$ and

$$
\Theta(z, t)=\frac{1}{\sqrt{4 \pi t}} \sum_{k=-\infty}^{+\infty} e^{-(z+2 k \pi)^{2} / 4 t}
$$

is the theta-function.

Denoting $d(x, y)$ by $\theta$ and using (5.13) and (5.12), one gets

$$
\begin{aligned}
& R(x, y ; \lambda-1)=\int_{0}^{+\infty} e^{\lambda t} e^{-t} H(x, y ; t) d t \\
& \quad=\frac{1}{4 \pi} \frac{1}{\sin \theta}\left(-\sum_{k<0} e^{(\theta+2 k \pi) \sqrt{-\lambda}}+\sum_{k \geq 0} e^{-(\theta+2 k \pi) \sqrt{-\lambda}}\right) \\
& \quad=\frac{1}{4 \pi} \frac{1}{\sin \theta} \frac{1}{1-e^{-2 \pi \sqrt{-\lambda}}}\left[-e^{-2 \pi \sqrt{-\lambda}} e^{\theta \sqrt{-\lambda}}+e^{-\theta \sqrt{-\lambda}}\right] \\
& \quad=\frac{1}{4 \pi \theta}-\frac{1}{4 \pi} \frac{1+e^{-2 \pi \sqrt{-\lambda}}}{1-e^{-2 \pi \sqrt{-\lambda}}} \sqrt{-\lambda}+o(1)
\end{aligned}
$$

as $\theta \rightarrow 0$, which implies the Lemma.

Example 2 (flat $3 d$-tori). Let $\{\mathbf{A}, \mathbf{B}, \mathbf{C}\}$ be a basis of $\mathbb{R}^{3}$ and let $T^{3}$ be the quotient of $\mathbb{R}^{3}$ by the lattice $\left\{m \mathbf{A}+n \mathbf{B}+l \mathbf{C}:(m, n, l) \in \mathbb{Z}^{3}\right\}$ provided with the usual flat metric.

Note that the free resolvent kernel in $R^{3}$ is

$$
\frac{e^{-\sqrt{-\lambda}\|x-y\|}}{4 \pi\|x-y\|}
$$


and, therefore,

$$
R(x, y ; \lambda)=\frac{e^{-\sqrt{-\lambda}\|x-y\|}}{4 \pi\|x-y\|}+\frac{1}{4 \pi} \sum_{(m, n, l) \in \mathbb{Z}^{3} \backslash(0,0,0)} \frac{e^{-\sqrt{-\lambda}\|x-y+m \mathbf{A}+n \mathbf{B}+l \mathbf{C}\|}}{\|x-y+m \mathbf{A}+n \mathbf{B}+l \mathbf{C}\|} .
$$

From (5.15) it follows that

$$
\begin{aligned}
F(\lambda) & =\frac{1}{4 \pi} \sqrt{-\lambda}-\frac{1}{4 \pi} \sum_{(m, n, l) \in \mathbb{Z}^{3} \backslash(0,0,0)} \frac{e^{-\sqrt{-\lambda}\|m \mathbf{A}+n \mathbf{B}+l \mathbf{C}\|}}{\|m \mathbf{A}+n \mathbf{B}+l \mathbf{C}\|} \\
& =\frac{1}{4 \pi} \sqrt{-\lambda}+O\left(|\lambda|^{-\infty}\right)
\end{aligned}
$$

as $\lambda \rightarrow-\infty$

Remark 3. It should be noted that explicit expressions for $\operatorname{det}^{*} \Delta$ in case $X=S^{3}$ and $X=T^{3}$ are given in [13] and [6].

\section{Acknowledgment}

The work of T.A. was supported by FQRNT and the research of A.K. was supported by NSERC.

\section{References}

[1] S. Albeverio, F. Gesztesy, R. Hoegh-Krohn and H. Holden, Solvable models in quantum mechanics, AMS 2005.

[2] A. Erdélyi, and H. Bateman, Tables of integral transforms, vol 2, McGraw-Hill, New York, 1954.

[3] Yves Colin De Verdiere, Pseudo-Laplaciens. I, Annales de l'institut Fourier, tome 32, N3 (1982), $275-286$.

[4] J. Cheeger and M. Taylor, On the diffraction of waves by conical singularities. I, Commun. Pure Appl. Math., 35(3) (1982), 275-331.

[5] Fay, D. John, Kernel functions, analytic torsion, and moduli spaces, Memoirs of the AMS 464 (1992)

[6] K. Furutani and S. de Gosson, Determinant of Laplacians on Heisenberg manifolds, J. Geom. Phys., 48 (2003), 438-479.

[7] L. Hillairet and A. Kokotov, Krein formula and S-matrix for euclidean surfaces with conical singularities, J. Geom. Anal, 2012, to appear, arXiv:1011.5034v1

[8] K. Kirsten, P. Loya and J. Park, Exotic expansions and pathological properties of $\zeta$-functions on conic manifolds, J. Geom. Anal. 18 (2009), 835-888.

[9] K. Kirsten, P. Loya and J. Park, The very unusual properties of the resolvent, heat kernel, and zeta-function for the operator $-\frac{d^{2}}{d r^{2}}-1 /\left(4 r^{2}\right)$, J. Math. Phys., 47 (2006)

[10] P. Loya, P. McDonald and J. Park, Zeta regularized determinants for conic manifolds, J. Funct. Anal. 242(N1) (2007), 195-229.

[11] D.B. Ray and I.M. Singer, Analytic torsion for complex manifolds, Ann. Math. 98 (1973), 154-177.

[12] S. Minakshisundaram and A. Pleijel, Some properties of the eigenfunctions of the Laplaceoperator on Riemannian manifolds, Can. J. Math. 1 (1949), 242-256.

[13] H. Kumagai, The determinant of the Laplacian on the $n$-sphere, Acta Arithmatic., XCL.3 (1999)

[14] D.B. Ray and I.M. Singer, Analytic torsion for complex manifolds, Ann. of Math. 98(N1) (1973), 154-177.

[15] H. Ueberschaer, The trace formula for a point scatterer on a compact hyperbolic surface, arXiv: $1109.4329 \mathrm{v} 2$ 
Department of Mathematics and Statistics, Concordia University, 1455 de Maisonneuve Blvd., West Montreal, Quebec H3G 1M8, Canada

E-mail address: tayeb.aissiou@mail.mcgill.ca

UMR CNRS, 6629-Université de NAntes, 2 RUe De LA Houssinière, BP 92 208, F-44 322 Nantes Cedex 3, France

E-mail address: Luc.Hillairet@math.univ-nantes.fr

Department of Mathematics and Statistics, Concordia University, 1455 de Maisonneuve Blvd. West, Montreal, Quebec H3G 1M8 Canada

E-mail address: alexey@mathstat.concordia.ca 